\title{
The relationship between serum fibrosis markers and restrictive ventricular filling in patients with heart failure with reduced ejection fraction: A technetium-99m radionuclide ventriculography study
}

\author{
Yen-Tin Lin ${ }^{1, *}$, Yen-Hung Lin ${ }^{2,5},{ }^{*}$, Xue-Ming $\mathbf{W u}^{1}{ }$, Chi-Lun Ko ${ }^{3}$, Ruoh-Fang Yen ${ }^{3}$, \\ Ying-Hsein Chen ${ }^{2}$, Ron-Bin $\mathrm{Hsu}^{4}$, Chi-Ming Lee ${ }^{2}$, Shoei-Shen Wang ${ }^{4}$, Ming-Fong \\ Chen ${ }^{2,6}$ and Yen-Wen $\mathbf{W u}^{2,3,7,8}$ \\ ${ }^{1}$ Department of Internal Medicine, Taoyuan General Hospital, Ministry of Health and Welfare, Taoyuan, Taiwan \\ 2 Department of Internal Medicine, National Taiwan University Hospital and National Taiwan University College of Medicine, \\ Taipei, Taiwan \\ ${ }^{3}$ Department of Nuclear Medicine, National Taiwan University Hospital and National Taiwan University College of Medicine, \\ Taipei, Taiwan \\ ${ }^{4}$ Department of Surgery, National Taiwan University Hospital and National Taiwan University College of Medicine, Taipei, \\ Taiwan \\ ${ }^{5}$ Department of Medicine, Beth Israel Deaconess Medical Center/Harvard Medical School, Boston, Massachusetts, USA \\ ${ }^{6}$ Cardiovascular Research Laboratory, Cardiovascular Center, Clinical Outcome Research and Training Center, Big Data \\ Center, China Medical University Hospital, China Medical University, Taichung, Taiwan \\ ${ }^{7}$ Cardiology Division of Cardiovascular Medical Center, Department of Nuclear Medicine, Far Eastern Memorial Hospital, New \\ Taipei City, Taiwan \\ ${ }^{8}$ National Yang-Ming University School of Medicine, Taipei, Taiwan \\ * These authors have contributed equally to this work \\ Correspondence to: Yen-Wen Wu, email:wuyw0502@gmail.com
}

Keywords: fibrosis; restrictive filling; ventriculography

Received: October 04, $2016 \quad$ Accepted: November 24, 2016

Published: December 04, 2016

\section{ABSTRACT}

Myocardial fibrosis leads to a restrictive diastolic filling pattern of the left ventricle which is associated with a poor prognosis in patients with heart failure. We investigated the relationship between cardiac fibrosis and restrictive filling pattern of the left ventricle measured by $\mathrm{Tc} 99 \mathrm{~m}$ left ventriculography in patients with chronic symptomatic heart failure. Serum cardiac extracellular matrix markers including type I and III aminoterminal propeptide of procollagen (PINP and PIIINP), matrix metalloproteinase-2,9 (MMP-2,9), and tissue inhibitor of MMP-1 (TIMP-1) were analyzed. Fifty-one (39 males) patients were enrolled. Their median age was $\mathbf{5 1 . 8}$ years, and median left ventricular ejection fraction was $31.9 \%$. Time to peak filling rate of the left ventricle was significantly correlated with serum levels of the three cardiac extracellular matrix markers (TIMP-1, PIIINP, and MMP-2). The patients with a restrictive diastolic filling pattern of the left ventricle (time to peak filling rate $\leq 154$ ms) had significantly higher levels of these extracellular matrix markers. In receiver operating characteristic curve analysis, areas under the curve of PIIINP, TIMP-1, and MMP-2 were $0.758,0.695$, and 0.751 to predict the presence of a restrictive pattern. In $\mathrm{C}$-statistics, all three cardiac extracellular matrix markers significantly increased the area under the curve after adding creatinine. In net reclassification improvement and integrated discrimination improvement models, PIIINP and MMP-2 significantly improved the predictive power of age, creatinine and brain natriuretic peptide. In conclusion, serum extracellular matrix markers are significantly correlated with restrictive diastolic filling pattern of the left ventricle in patients with heart failure. 


\section{INTRODUCTION}

Heart failure (HF) is a major health problem affecting more than 23 million patients worldwide [1]. Despite remarkable improvements in medical treatment in recent decades, the prognosis of patients with HF remains poor [2].

Systolic function has been reported to be a prognostic predictor in HF with reduced ejection fraction (HFrEF) [3]. However, compared to the New York Heart Association functional classification, left ventricular ejection fraction (LVEF) has been reported to be a less reliable marker, especially in patients with atrial fibrillation $[4,5]$. Beyond systolic function, abnormal diastolic function is also an important factor. Patients with HFrEF and concomitant diastolic dysfunction have been reported to have worse clinical outcomes, and especially those with severe impairment of diastolic function such as a restrictive filling pattern in mitral flow [6]. Many tools can estimate diastolic function, including echocardiography, cardiac magnetic resonance imaging (MRI), electrocardiography-gated myocardial perfusion single photon emission computed tomography (SPECT), and technetium 99m (Tc99m) left ventriculography. Echocardiography is simple, non-invasive and costeffective, however it is limited by alterations in loading conditions, operator dependency, and echo window. Tc99m left ventriculography has recently been reported to be a useful method to assess LV diastolic dysfunction due to more stable parameters and because it is independent of the influence of systolic function, age and sex [7]. However, data on LV diastolic function in patients with HF are limited.

Left ventricle remodeling plays a critical role in the progression of HF. Several serum markers of cardiac extracellular matrix (ECM) have been shown to play an important role in myocardial remodeling in various types of heart disease in patients with HFrEF and in animal models [8] [9] [10]. Restrictive LV filling develops as a consequence of myocardial remodeling and cardiac fibrosis, and it has been reported to be closely associated with clinical outcomes [11, 12]. Detecting restrictive LV filling is therefore important, however it is highly dependent on the imaging tool.

Several cardiac ECM markers including type III aminoterminal propeptide of procollagen (PIIINP), matrix metalloproteinases (MMPs), and tissue inhibitor of metalloproteinase-1 (TIMP-1) have recently been shown to be of prognostic value in patients with HF [13] [1417]. These markers reflect the collagen turnover process in the myocardium and are useful to evaluate fibrosis in the myocardium. Therefore, cardiac ECM markers could potentially be used to detect LV restrictive filling.

In this study, we investigated the relationships between serum markers of cardiac ECM and LV restrictive filling using Tc99m left ventriculography in patients with HFrEF.

\section{MATERIALS AND METHODS}

\section{Patients}

We studied 51 patients with chronic HF secondary to $\mathrm{LV}$ systolic dysfunction (LVEF $\leq 45 \%$ measured using Tc99m left ventriculography) who regularly visited the HF clinics at National Taiwan University Hospital. The clinical history of all patients was recorded, and they all received a full examination by a cardiologist. Demographic data including sex, age, current medications, functional status, and cardiovascular risk factors were recorded. Venous blood samples were collected in serum separation tubes after overnight fasting. After clotting and centrifugation, the serum was collected and stored at $-60^{\circ} \mathrm{C}$ until analysis. In echocardiography, LVEF was measured via an apical 4-chamber view (area-length method) according to the procedures of the American Society of Echocardiography [18]. The study was approved by the Ethics Committee of National Taiwan University Hospital, and all of the patients provided written informed consent.

\section{Diastolic function assessment}

We assessed LV diastolic function using Tc99m left ventriculography. Peak-filling rate (PFR) was defined as the greatest filling rate in early diastole, and corresponded to the peak value of the first derivative of the diastolic portion of the time-activity curve. The filling was normalized to end-diastolic volume (EDV), and thus the unit for PFR was EDV/s. The time to peak filling rate (TPFR), expressed in milliseconds, was defined as the interval between end-systole and PFR.

The normal PFR of the left ventricle has been reported to be $2.2+/-0.6 \mathrm{EDV} / \mathrm{sec}$, and $198+/-22 \mathrm{~ms}$ for TPFR [14-17, 19]. By applying a 2 -standard deviation cutoff point to the mean values of TPFR, the threshold for a restrictive pattern of $\mathrm{LV}$ filling was defined as a TPFR $\leq 154 \mathrm{~ms}$

\section{Laboratory analysis}

Serum levels of brain natriuretic peptide (BNP) were measured using an enzyme immunoassay kit (BNP-32, Phoenix pharmaceuticals, Belmont, USA). The intra- and inter-assay variations were $<5 \%$ and $<$ $14 \%$, respectively. Serum levels of type I aminoterminal propeptide of procollagen (PINP) were measured using a rapid equilibrium radioimmunoassay kit (No. 67034, 
Table 1: Demographic data between patients of restrictive and non-restrictive diastolic function of $L V$

\begin{tabular}{|c|c|c|c|c|}
\hline & Total population & $\begin{array}{l}\text { Restrictive } \\
\text { function }(n=21)\end{array} \quad$ diastolic & $\begin{array}{l}\text { Non-restrictive diastolic } \\
\text { function }(n=30)\end{array}$ & $p$-value \\
\hline Age & $51.8(47.2-73.0)$ & $68.2(48.4,78.3)$ & $50.4(42.8,64.8)$ & 0.028 \\
\hline Gender, M/F & $39 / 12$ & $15 / 6$ & $24 / 6$ & 0.478 \\
\hline Body mass index, $\mathrm{Kg} / \mathrm{m}^{2}$ & $25.1(22.3,29.0)$ & $25.2(21.9,29.4)$ & $25.0(22.7,29.0)$ & 0.969 \\
\hline LVEF by echocardiography, $\%$ & $36(26,41)$ & $35(24,42)$ & $37(26,43)$ & 0.730 \\
\hline $\begin{array}{l}\text { NYHA functional classification, } \\
\text { I/II/III/IV }\end{array}$ & $8 / 27 / 14 / 2$ & $3 / 10 / 7 / 1$ & $5 / 17 / 7 / 1$ & 0.861 \\
\hline \multicolumn{5}{|l|}{ Etiology for heart failure } \\
\hline Ischemic & $21(41 \%)$ & $9(43 \%)$ & $12(40 \%)$ & 0.838 \\
\hline Non-Ischemic & $30(59 \%)$ & $12(57 \%)$ & $18(60 \%)$ & \\
\hline \multicolumn{5}{|l|}{ Co-morbidity } \\
\hline Hypertension & $22(43 \%)$ & $10(48 \%)$ & $12(40 \%)$ & 0.589 \\
\hline Diabetes Mellitus & $13(25 \%)$ & $6(29 \%)$ & $7(23 \%)$ & 0.673 \\
\hline Atrial fibrillation & $14(27 \%)$ & $7(33 \%)$ & $7(23 \%)$ & 0.431 \\
\hline \multicolumn{5}{|l|}{ Biochemistry } \\
\hline Hemoglobin, g/dL & $14.2(12.9,15.5)$ & $13.9(12.4,15.6)$ & $14.2(13.3,15.6)$ & 0.406 \\
\hline Triglyceride, $\mathrm{mg} / \mathrm{dl}$ & $146(85,216)$ & $112(83,179)$ & $165(87,280)$ & 0.203 \\
\hline Total cholesterol, mg/dl & $156(186,212)$ & $171(156,215)$ & $199(158,212)$ & 0.328 \\
\hline Fasting glucose, $\mathrm{mg} / \mathrm{dl}$ & $100(88,136)$ & $100(87,135)$ & $100(90,142)$ & 0.762 \\
\hline Creatinine, $\mathrm{mg} / \mathrm{dL}$ & $1.2(1.0,1.4)$ & $1.3(1.1,1.5)$ & $1.2(1.0,1.4)$ & 0.268 \\
\hline BNP, pg/mL & $2061(1430,2520)$ & $2263(1413,2780)$ & $1957(1575,2435)$ & 0.579 \\
\hline \multicolumn{5}{|l|}{ Medications } \\
\hline ACEI / ARB & $28(55 \%)$ & $10(48 \%)$ & $18(60 \%)$ & 0.382 \\
\hline$\beta$-blocker & $34(67 \%)$ & $14(67 \%)$ & $20(67 \%)$ & 1.000 \\
\hline Loop agents & $35(69 \%)$ & $17(81 \%)$ & $18(60 \%)$ & 0.112 \\
\hline \begin{tabular}{|l|} 
Digoxin \\
\end{tabular} & $28(55 \%)$ & $13(62 \%)$ & $15(50 \%)$ & 0.400 \\
\hline Spironolactone & $20(39 \%)$ & $9(43 \%)$ & $11(37 \%)$ & 0.656 \\
\hline Statin & $7(14 \%)$ & $3(14 \%)$ & $4(13 \%)$ & 0.923 \\
\hline
\end{tabular}

Abbreviations: $\mathrm{BNP}=$ Brain natriuretic peptide; $\mathrm{ACE}-\mathrm{I}=$ angiotensin converting enzyme inhibitor; $\mathrm{ARB}=$ angiotensin receptor blocker; $\mathrm{LVEF}=$ left ventricular ejection fraction; $\mathrm{MMP}=$ matrix metalloproteinase; NYHA Fc= New York Heart Association functional classification.

Orion Diagnostica, Espoo, Finland). The intra- and inter-assay variations were both $<7 \%$, and the detection limit was $2 \mu \mathrm{g} / \mathrm{l}$. Serum PIIINP was determined using a coated-tube radioimmunoassay method (No. 68570, Orion Diagnostica, Espoo, Finland). The intra- and interassay variations of serum PIIINP were both $<5 \%$, and the detection limit was $0.3 \mu \mathrm{g} / \mathrm{l}$. TIMP-1 was measured using an ELISA kit (DTM100, R \& D Systems, Minneapolis, USA). The intra- and inter-assay variations of serum TIMP-1 were both $<5 \%$, and the detection limit was 0.08 $\mathrm{ng} / \mathrm{ml}$. Serum MMP-2 was measured using an ELISA kit (DMP200, R \& D Systems, Minneapolis, USA). The intraand inter-assay variations of this method were $<6 \%$ and $<8 \%$, respectively, with a detection limit of $0.16 \mathrm{ng} / \mathrm{ml}$. MMP-9 was also measured using an ELISA kit (DMP900, R \& D Systems, Minneapolis, USA). The detection limit was $0.156 \mathrm{ng} / \mathrm{mL}$, and the intra- and inter-assay variations were $<3 \%$ and $<8 \%$, respectively.

\section{Statistical analysis}

Continuous data were expressed as median value and interquartile range. Comparisons between groups for continuous data were performed using the MannWhitney U-test, and differences between proportions were assessed using the chi-square or Fisher's exact test. Spearman's non-parametric correlation test was used to analyze associations between ventricular diastolic function and serum fibrosis markers. Linear regression analysis was used to examine associations between serum fibrosis markers and LV restrictive diastolic function. The serum cardiac ECM markers were log-transformed due to nonnormality as detected using the Kolmogorov-Smirnov test before being entered into the linear regression model. To compare the ability of serum cardiac ECM markers to predict the patients with a restrictive pattern in diastolic 
Table 2: Median level of serum fibrosis markers and Tc99m radionuclide ventriculography parameter $(\mathrm{n}=51)$

\begin{tabular}{|l|l|l|l|l|}
\hline & Total & $\begin{array}{l}\text { Restrictive diastolic } \\
\text { function }(\mathbf{n}=\mathbf{2 1})\end{array}$ & $\begin{array}{l}\text { Non-restrictive diastolic } \\
\text { function }(\mathbf{n}=30)\end{array}$ & p-value \\
\hline Serum fibrosis markers & & & & \\
\hline PINP, $\mu \mathrm{g} / \mathrm{l}$ & $33.02(24.16-46.85)$ & $40.48(27.35,51.83)$ & $30.68(22.05,40.67)$ & 0.112 \\
\hline TIMP-1 ng/ml & $131.3(96.6-181.8)$ & $142.70(119.95,204.60)$ & $114.7(93.35,155,35)$ & 0.019 \\
\hline PIIINP, $\mu \mathrm{g} / \mathrm{l}$ & $6.4(4.64-7.21)$ & $7.03(6.43,9.60)$ & $5.13(4.29,6.87)$ & 0.002 \\
\hline MMP-2 ng/ml & $248.47(218.39-318.76)$ & $310.49(234.18,419.05)$ & $236.09(209.03,268.03)$ & 0.002 \\
\hline MMP-9 ng/ml & $58.1(38.5-100.7)$ & $52.70(32.10,104.50)$ & $59.15(39.77,100.65)$ & 0.688 \\
\hline $\begin{array}{l}\text { Technetium-99m radionuclide } \\
\text { ventriculography parameter }\end{array}$ & $31.9(21.7,38.9)$ & & & \\
\hline LVEF, \% & $1.50(1.07,1.88)$ & $1.57(1.22,2.14)$ & $1.44(1.04,1.79)$ & 0.121 \\
\hline LV-PFR, EDV/sec & $177(118,348)$ & $101(64,141)$ & $250(196,416)$ & 0.461 \\
\hline LV-TPFR, ms & $43.0(31.0,48.8)$ & $42.3(31.5,47.3)$ & $43.0(30.5,52.1)$ & $<0.001$ \\
\hline RVEF, \% & $2.06(1.58,2.80)$ & $2.10(1.71,3.12)$ & $1.95(1.53,2.54)$ & 0.660 \\
\hline RV-PFR, EDV/sec & $145(80,308)$ & $71(59,147)$ & $222.5(142,399)$ & 0.394 \\
\hline RV-TPFR, ms & & & $35.6(21.9,40.1)$ & \\
\hline
\end{tabular}

Abbreviations: $\mathrm{LVEF}=$ left ventricular ejection fraction; $\mathrm{LV}-\mathrm{PFR}=$ left ventricle-peak filling rate; $\mathrm{LV}-\mathrm{TPFR}=$ left ventricletime to peak filling rate; $\mathrm{RVEF}=$ right ventricular ejection fraction; RV-PFR= right ventricle-peak filling rate; $\mathrm{RV}-\mathrm{TPFR}=$ right ventricle-time to peak filling rate; $\mathrm{MMP}=$ matrix metalloproteinase; $\mathrm{NYHA} \mathrm{Fc}=$ New York Heart Association functional classification; PINP = type I aminoterminal propeptide of procollagen; PIIINP = type III aminoterminal propeptide of procollagen; TIMP = tissue inhibitor of metalloproteinase.

filling (TPFR $\leq 154 \mathrm{~ms}$ ), we used areas under the receiver operator characteristic (ROC) curve determined by logistic regression analysis. The optimal cutoff points of serum fibrosis markers were obtained from the Youden index.

We also used $C$-statistics to describe the discrimination of the models before and after adding serum cardiac ECM markers [20-22]. Net reclassification improvement (NRI) and integrated discrimination improvement (IDI) models were performed to assess improvements in risk prediction using two different logistic regression models [21]. All statistical analyses were performed using R software (http://www.r-project. org/) version 2.15.2. Statistical significance was set at $p$ $<0.05$.

\section{RESULTS}

\section{Patient characteristics}

Fifty-one (39 males and 12 females) patients were enrolled with a median age of 51.8 years, and median LVEF of $36.2 \%$ by echocardiography and $31.9 \%$ by Tc99m left ventriculography. Their median NYHA was 2 , with $8,27,14$, and 2 patients having a NYHA of I, II, III, and IV, respectively. The other demographic and biochemical data and medication history are shown in Table 1.

We divided the patients into two groups based on the presence of a restrictive pattern in Tc99m radionuclide ventriculography. The mean age was significantly older in the restrictive pattern group ( $p=0.028$ ), but there were no significant differences in the other demographic data (Table 1).

\section{Correlation between serum fibrosis markers and Tc99m radionuclide ventriculography parameters}

The mean level of serum fibrosis markers and Tc99m radionuclide ventriculography are shown in Table 2. TPFR of the left ventricle was significantly correlated with serum levels of TIMP-1 ( $\mathrm{r}=-0.337, p=$ $0.016)$, PIIINP ( $\mathrm{r}=-0.445, p=0.001)$, and MMP-2 ( $\mathrm{r}=$ $-0.391, p=0.05)$, whereas TPFR of the right ventricle was significantly correlated with serum PIIINP levels $(\mathrm{r}=$ $-0.320, p=0.022$ ) (Table 3).

\section{Relationship between the presence of a restrictive pattern in Tc99m radionuclide ventriculography and serum fibrosis markers}

The patients with a restrictive LV pattern in Tc99m radionuclide ventriculography had significantly higher levels of serum fibrosis markers including TIMP-1 ( $p=$ $0.019)$, PIIINP ( $p=0.002)$, and MMP-2 $(p=0.002)$ (Table $2)$. The relationship between serum fibrosis markers and the presence of a restrictive pattern were still significant after adjusting for age and sex (Table 4). The relationship between PIIINP or MMP-2 and the presence of a restrictive pattern remained significant after adjusting for age, sex, and serum creatinine level (Table 4). 
Table 3: Spearman rank correlation between the parameters of Tc99m radionuclide ventriculography \& serum fibrosis markers

\begin{tabular}{|l|c|c|c|c|c|c|}
\hline & RNA-LVEF & LV-PFR & LV-TPFR & RNA-RVEF & RV-PFR & RV-TPFR \\
\hline \multirow{2}{*}{ PINP } & $\mathrm{r}=-0.034$ & $\mathrm{r}=0.056$ & $\mathrm{r}=-0.220$ & $\mathrm{r}=-0.014$ & $\mathrm{r}=0.106$ & $\mathrm{r}=-0.186$ \\
& $\mathrm{p}=0.813$ & $\mathrm{p}=0.696$ & $\mathrm{p}=0.121$ & $\mathrm{p}=0.920$ & $\mathrm{p}=0.461$ & $\mathrm{p}=0.191$ \\
\hline \multirow{2}{*}{ PIIINP } & $\mathrm{r}=0.124$ & $\mathrm{r}=0.283$ & $\mathrm{r}=-0.445$ & $\mathrm{r}=-0.110$ & $\mathrm{r}=0.214$ & $\mathrm{r}=-0.320$ \\
& $\mathrm{p}=0.387$ & $\mathrm{p}=0.044$ & $\mathrm{p}=0.001$ & $\mathrm{p}=0.441$ & $\mathrm{p}=0.131$ & $\mathrm{p}=0.022$ \\
\hline \multirow{2}{*}{ TIMP-1 } & $\mathrm{r}=0.109$ & $\mathrm{r}=0.138$ & $\mathrm{r}=-0.337$ & $\mathrm{r}=-0.066$ & $\mathrm{r}=-0.029$ & $\mathrm{r}=-0.179$ \\
& $\mathrm{p}=0.445$ & $\mathrm{p}=0.335$ & $\mathrm{p}=0.016$ & $\mathrm{p}=0.644$ & $\mathrm{p}=0.842$ & $\mathrm{p}=0.209$ \\
\hline \multirow{2}{*}{ MMP-2 } & $\mathrm{r}=-0.049$ & $\mathrm{r}=0.099$ & $\mathrm{r}=-0.391$ & $\mathrm{r}=-0.210$ & $\mathrm{r}=0.077$ & $\mathrm{r}=-0.231$ \\
& $\mathrm{p}=0.732$ & $\mathrm{p}=0.491$ & $\mathrm{p}=0.005$ & $\mathrm{p}=0.140$ & $\mathrm{p}=0.589$ & $\mathrm{p}=0.102$ \\
\hline \multirow{2}{*}{ MMP-9 } & $\mathrm{r}=-0.001$ & $\mathrm{r}=0.216$ & $\mathrm{r}=-0.041$ & $\mathrm{r}=0.013$ & $\mathrm{r}=0.154$ & $\mathrm{r}=0.066$ \\
& $\mathrm{p}=0.995$ & $\mathrm{p}=0.128$ & $\mathrm{p}=0.773$ & $\mathrm{p}=0.929$ & $\mathrm{p}=0.282$ & $\mathrm{p}=0.645$ \\
\hline
\end{tabular}

$\mathrm{LVEF}=$ left ventricular ejection fraction; $\mathrm{LV}-\mathrm{PFR}=$ left ventricle-peak filling rate; Abbreviations: $\mathrm{LV}-\mathrm{TPFR}=$ left ventricletime to peak filling rate; $\mathrm{RVEF}=$ right ventricular ejection fraction; RV-PFR= right ventricle-peak filling rate; $\mathrm{RV}-\mathrm{TPFR}=$ right ventricle-time to peak filling rate; $\mathrm{MMP}=$ matrix metalloproteinase; NYHA Fc= New York Heart Association functional classification; PINP = type I aminoterminal propeptide of procollagen; PIIINP = type III aminoterminal propeptide of procollagen; TIMP = tissue inhibitor of metalloproteinase.

Table 4: Logistic regression between serum fibrosis markers and left ventricle restrictive diastolic function.

\begin{tabular}{|l|l|l|l|l|l|l|}
\hline & & $\log$ PINP & $\log$ PIIINP & $\log$ TIMP-1 & $\log$ MMP-2 & $\log$ MMP-9 \\
\hline \multirow{3}{*}{ Model 1 } & OR value & 14.440 & 436.115 & 126.813 & 3086.568 & 1.162 \\
& $95 \%$ CI & $0.658-316.812$ & $5.328-35704$ & $2.566-6268.181$ & $12.220-779604$ & $0.206-6.563$ \\
& p value & 0.090 & 0.007 & 0.015 & 0.004 & 0.865 \\
\hline \multirow{3}{*}{ Model 2 } & OR value & 18.928 & 622.391 & 72.266 & 1849.149 & 3.025 \\
& $95 \%$ CI & $0.606-591.478$ & $5.168-74948$ & $1.091-4784.690$ & $4.333-789163$ & $0.412-22.233$ \\
& p value & 0.094 & 0.008 & 0.045 & 0.015 & 0.277 \\
\hline \multirow{3}{*}{ Model 3 } & OR value & 13.776 & 6424.241 & 103.644 & 2607.875 & 2.615 \\
& 95\% CI & $0.284-668.015$ & $11.759-3509696$ & $0.796-13503.066$ & $3.383-2151712$ & $0.357-19.130$ \\
& p value & 0.185 & 0.006 & 0.062 & 0.020 & 0.277 \\
\hline
\end{tabular}

Model 1 unadjusted.

Model 2 adjusted for age and sex.

Model 3 adjusted for age, sex, and serum creatinine level.

Abbreviations: OR = odds ratio; CI = confidence interval; PINP = type I aminoterminal propeptide of procollagen; PIIINP = type III aminoterminal propeptide of procollagen; TIMP = tissue inhibitor of metalloproteinase.

\section{Prediction of a restrictive pattern in diastolic filling by various cardiac ECM markers and clinical parameters}

To predict the patients with a restrictive pattern in diastolic filling (TPFR $\leq 154 \mathrm{~ms}$ ), ROC analysis showed an area under the curve (AUC) of 0.632 for PINP, 0.758 for PIIINP, 0.695 for TIMP, 0.751 for MMP-2, and 0.467 for MMP-9 (Figure 1). Using a level of PIIINP of 6.22 $\mathrm{mg} / \mathrm{L}$ as a cut-off point, the sensitivity, specificity, positive predictive rate, and negative predictive rate were $100 \%$, $67 \%, 55 \%$, and $100 \%$, respectively. The AUC of clinical parameters and BNP were 0.631 for age, 0.471 for male gender, 0.595 for serum creatinine level, and 0.565 for $\mathrm{BNP}$, respectively.

\section{Analysis of the predictive value of cardiac ECM markers by AUC, NRI and IDI models}

We further examine the ability of the three cardiac ECM markers (PIIINP, TIMP-1, and MMP-2) to predict the patients with a restrictive pattern in diastolic filling after adding two clinical parameters (age and sex) or BNP. In $C$-statistics, all three cardiac ECM markers significantly increased the AUC of creatinine (Table 5). Furthermore, after adding cardiac ECM markers, the AUC of the new ROC curve was above 0.9 in all three models. The combination of creatinine and PIIINP had the highest AUC of 0.944. In the NRI and IDI models, PIIINP and MMP-2 significantly improved the predictive power of both clinical parameters and BNP, and TIMP-1 significantly improved the predictive power of creatinine (Table 5). 
Table 5: Analysis of the predictive values of biomarkers by AUC, NRI, and IDI models

\begin{tabular}{|l|l|l|l|l|l|l|}
\hline & AUC & $\begin{array}{l}\text { AUC } \\
\boldsymbol{p} \text { value }\end{array}$ & NRI & $\begin{array}{l}\text { NRI } \\
\boldsymbol{p} \text { value }\end{array}$ & IDI & $\begin{array}{l}\text { IDI } \\
\boldsymbol{p} \text { value }\end{array}$ \\
\hline PIIINP & 0.758 & & & & & \\
\hline TIMP-1 & 0.695 & & & & & \\
\hline MMP-2 & 0.751 & & & & & \\
\hline Age & 0.631 & & & & & \\
\hline +PIIINP & 0.792 & 0.277 & 0.638 & 0.018 & 0.125 & 0.012 \\
\hline +TIMP-1 & 0.746 & 0.547 & 0.257 & 0.347 & 0.085 & 0.055 \\
\hline +MMP-2 & 0.774 & 0.371 & 0.810 & 0.002 & 0.112 & 0.017 \\
\hline Creatinine & 0.595 & & & & & \\
\hline +PIIINP & 0.944 & 0.003 & 1.124 & $<0.001$ & 0.268 & $<0.001$ \\
\hline +TIMP-1 & 0.901 & 0.015 & 0.771 & 0.002 & 0.151 & 0.008 \\
\hline +MMP-2 & 0.904 & 0.013 & 0.571 & 0.031 & 0.156 & 0.005 \\
\hline BNP & 0.565 & & & & & \\
\hline +PIIINP & 0.770 & 0.398 & 0.800 & 0.002 & 0.144 & 0.005 \\
\hline +TIMP-1 & 0.699 & 0.895 & 0.448 & 0.103 & 0.117 & 0.020 \\
\hline+ MMP-2 & 0.737 & 0.617 & 0.676 & 0.011 & 0.180 & 0.003 \\
\hline
\end{tabular}

BNP, Brain natriuretic peptide; LVEF, left ventricular ejection fraction; MMP, matrix metalloproteinase; PIIINP, type III aminoterminal propeptide of procollagen; TIMP, tissue inhibitor of metalloproteinase; AUC, area under the curve; NRI, Net reclassification improvement; IDI, integrated discrimination improvement.

\section{DISCUSSION}

The major findings of this study are: 1) serum ECM markers including TIMP-1, PIIINP, and MMP-2 were significantly correlated to LV TPFR; 2) the patients with a restrictive pattern of LV diastolic function had higher
TIMP-1, PIIINP, and MMP-2 levels; 3) the results were still significant after adjusting for age and sex; 4) cardiac ECM markers significantly improved the power of the clinical parameters to predict the patients with a restrictive pattern in diastolic filling; and 5) the combination of PIIINP and creatinine had greatest power (AUC 0.944) to

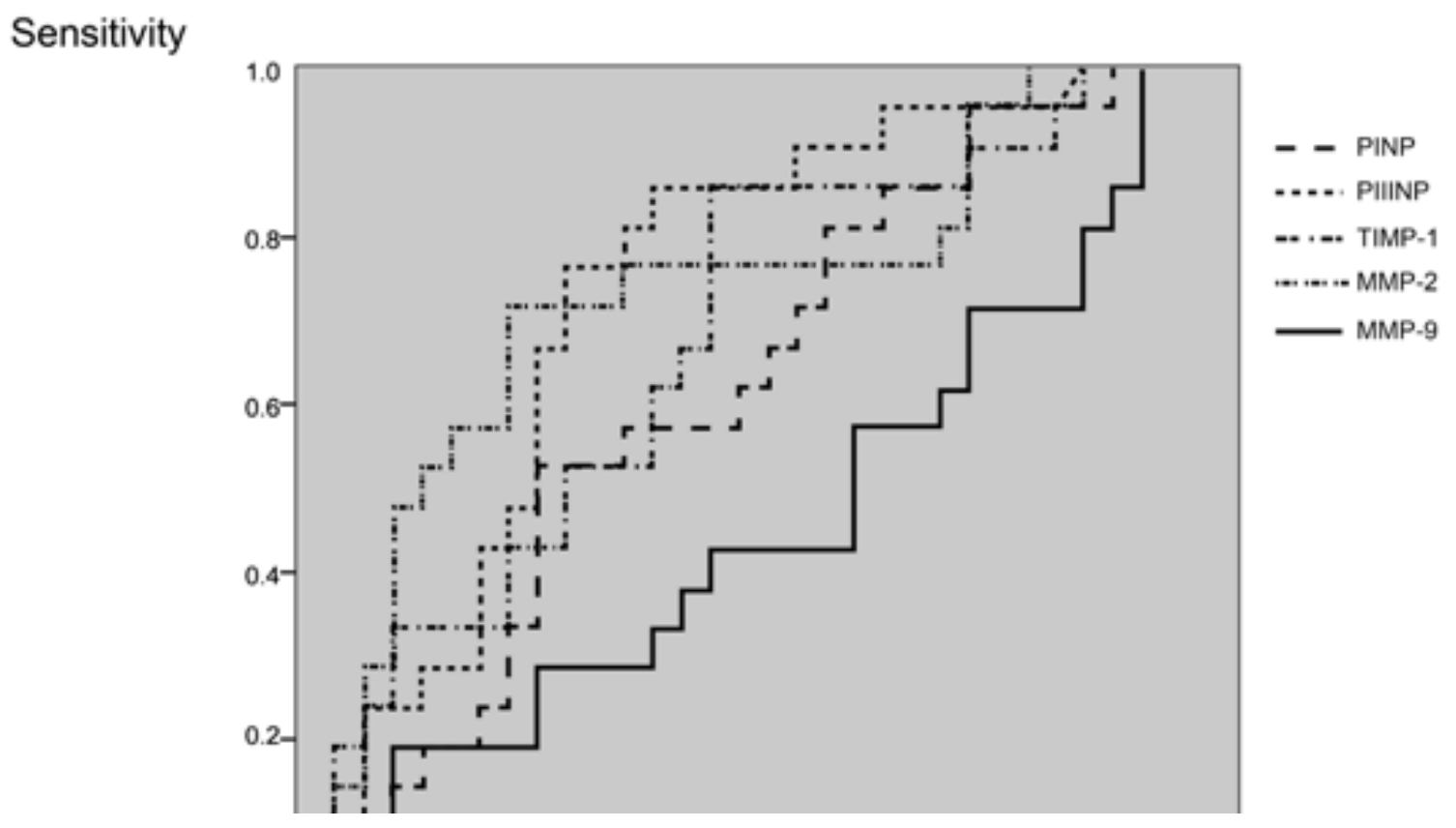

Figure 1: Analysis of the discrimination power of the two group by receiver operating characteristic curve analysis. The areas under the curve of PINP, PIIINP, TIMP-1, MMP-2, and MMP-9 were $0.632,0.758,0.695,0.751,0.467$, respectively. 
predict the patients with a restrictive pattern in diastolic filling. These findings not only reconfirmed the association between LV restrictive filling and cardiac ECM turnover, but also showed the potential of cardiac ECM markers as a useful tool to detect LV restrictive filling.

ECM turnover regulates a fine balance between synthesis and degradation [10, 23], and altering the balance of ECM markers can lead to cardiac fibrosis [17]. Cardiac fibrosis provides substrate for arrhythmia which can lead to sudden cardiac death. In addition, the worsening of cardiac diastolic dysfunction can also result in the progression of HF [24]. Therefore, measuring serum ECM markers may be a simple and reliable method to evaluate the degree of cardiac fibrosis [24]. Furthermore, clinical studies have shown that cardiac ECM markers can provide useful information on clinical symptoms, outcomes, and arrhythmia in patients with HFrEF [25].

In the present study, PIIINP had the best AUC. Both collagen types I and III are present in normal and diseased myocardial tissue. PINP and PIIINP are by-products of collagen type I or III synthesis, and therefore they are considered to be markers of collagen biosynthesis [26]. PIIINP is more specific to tissue in patients with cardiac disease, even though type I collagen is more abundant in the myocardium [25]. This is probably the reason why PIIINP had a better AUC that PINP in our analysis. PIIINP has also shown to have a better association with clinical outcomes than PINP in other studies [27].

MMPs are a family of enzymes that contribute to extracellular remodeling in several disease states. In addition, a family of inhibitors called tissue inhibitors of MMPs (TIMPs) have been shown to exist and to tightly regulate MMP activity. TIMP-1 has been shown to be highly associated with collagen accumulation and cardiac diastolic function $[28,29]$. Both MMP-2 and TIMP-1 have been demonstrated to have a prognostic role in patients with HF [30] [31]. In the present study, the AUC of MMP2 was 0.751 , which is very close to the AUC of PIIINP (0.758). We previously showed that MMP-2 was the best ECM marker to predict clinical outcomes [31]. Therefore, it is not surprising that MMP-2 was highly associated with LV restrictive filling in the present study.

In the Val-HeFT trial, a decrease in LVEF was associated with an increase in all-cause mortality at 23 months [32]. In addition, Solomon et al. reported a $39 \%$ increase in all-cause mortality in patients with $\mathrm{HFrEF}$ for every $10 \%$ reduction in ejection fraction below $45 \%$ [33]. However, relying on a univariate predictor such as LVEF to predict the prognosis is not sufficient, and patients with HFrEF and diastolic dysfunction have a lower survival rate. Both a deceleration time $\leq 140 \mathrm{~ms}$ and early to late flow velocity of mitral inflow $>1$ mean have been shown to be powerful independent predictors of mortality according to studies which evaluated mitral inflow patterns by Doppler echocardiography [34] [35]. In addition, Temporelli et al. reported that after optimal medical treatment, the patients' response to medication with reversal of the restrictive pattern at 6 months resulted in a lower cardiac mortality rate and fewer events requiring hospitalization than those with persistence of the restrictive pattern [36]. Patients with a persistent LV restrictive filling pattern have also been reported to have a significantly lower survival rate compare to patients with a reversible LV filling pattern [37].

Diastolic function can be estimated using echocardiography, cardiac MRI, and gate myocardial perfusion SPECT. Echocardiography is a simple, noninvasive and cost-effective tool, however alterations in loading conditions, operator technique, and the limitation of an acoustic window can all affect interpretation of the results. Although more expensive than echocardiography, cardiac MRI provides excellent spatial resolution and more precise parameters to assess diastolic function [38], however it takes time and needs the patient's cooperation. Moreover, patients with intra-cardiac devices are not candidates for MRI, and gadolinium injections to detect myocardial fibrosis may not be tolerable in patients with chronic kidney disease [38] [39].

Gated myocardial perfusion SPECT is widely used to assess perfusion abnormalities, LV systolic function and volume. First-pass and gated blood-pool scintigraphy including Tc99m left ventriculography are useful methods to assess LV diastolic dysfunction due to more stable parameters and independence from the influence of systolic function, age and sex [7]. Prolonged LV PFR and TPFR indicates impairment of diastolic function. After analyzing 90 patients with normal exercise gated myocardial perfusion SPECT, abnormal PFR and the threshold for abnormal TPFR were derived by 2 standard deviations to the mean values (PFR $<1.71 \mathrm{EDV} / \mathrm{s}$ and TPFR $>216.7 \mathrm{~ms}$ ) [7]. Compared to PFR, TPFR appears to be a more useful parameter to assess diastolic function independently of systolic function, heart rate and age [7]. For an extremely short TPFR, however, no previous studies have assessed restrictive diastolic function by Tc99m left ventriculography. By applying a 2-standard deviation cutoff to the mean value of TPFR, we defined a TPFR $<154 \mathrm{~ms}$ as a restrictive pattern of LV diastolic function. In this study, TPFR was negatively correlated with cardiac ECM markers including PIIINP, MMP-2 and TIMP-1. In the patients with a LV restrictive pattern, the cardiac ECM markers were significantly elevated. Furthermore, using the correlations between cardiac serum markers and restrictive function, we determined the threshold of cardiac ECM markers. This suggests that it will be possible to detect restrictive LV filling in failing hearts in the near future through simple blood testing.

There are several limitations to this study. First, the number of enrolled patients was relatively small, and may not be sufficient to provide definitive evidence. However, our findings may be sufficient to prompt further research. Second, we focused on HFrEF, and we are not sure 
whether similar results would be found in patients with HF with preserved ejection fraction. This could form the basis of further studies. Third, no histological evidence was available to prove myocardial remodeling and to assess the extent of fibrosis. Elevated levels of serum cardiac ECM markers may not present to the same degree over the myocardium. However, without clinical indications, it is not ethical to perform endomyocardial biopsies in these patients. Fourth, there is currently no general consensus on the definition of LV diastolic dysfunction and restrictive filling pattern in radionuclide ventriculography. Therefore, we used our own definition of "restrictive pattern" in this study. Further large-scale clinical studies and consensuses are needed to define the criteria of diastolic dysfunction and restrictive pattern in radionuclide ventriculography. Finally, a longer period of follow-up may be necessary to ascertain the clinical prognostic value of each biomarker and to differentiate outcomes between the patients with and without a restrictive LV filling pattern.

In conclusion, PIINP, MMP-2, and TIMP-1 are significantly associated with a shortened TPFR in Tc99m left ventriculography and the presence of restrictive LV filling in patients with HFrEF. These serum markers have the potential to detect the presence of $\mathrm{LV}$ restriction in patients with $\mathrm{HFrEF}$.

\section{ACKNOWLEDGMENTS}

The authors would like to thank the staff of the Second Core Lab of Department of Medical Research in National Taiwan University Hospital for their great support. This study was supported by the Department of Health, Executive Yuan, R.O.C. (PTH105-11, and PTH 105-08), National Taiwan University Hospital (NTUH 105-S3044, and UN105-060), and the Ministry of Science and Technology (MOST 101-2314-B-418-012-MY3, MOST 103-2220-E-002-011, MOST 104-2314-B-418008, MOST 105-2628-B-418-002-MY2, and MOST $105-$ 2314-B-002 -122 -MY3). The funders had no role in study design, data collection and analysis, decision to publish, or preparation of the manuscript.

\section{CONFLICTS OF INTEREST}

The authors report no conflicts of interest.

\section{REFERENCES}

1. Dunlay SM, Roger VL. Understanding the Epidemic of Heart Failure: Past, Present, and Future. Curr Heart Fail Rep. 2014; 11(4):404-15.

2. Go AS, Mozaffarian D, Roger VL, Benjamin EJ, Berry JD, Blaha MJ, Dai S, Ford ES, Fox CS, Franco S, Fullerton HJ, Gillespie C, Hailpern SM, et al. Heart disease and stroke statistics-2014 update: a report from the American Heart
Association. Circulation. 2014; 129:e28-e292.

3. Curtis JP, Sokol SI, Wang Y, Rathore SS, Ko DT, Jadbabaie F, Portnay EL, Marshalko SJ, Radford MJ, Krumholz HM. The association of left ventricular ejection fraction, mortality, and cause of death in stable outpatients with heart failure. J Am Coll Cardiol. 2003; 42(4):736-742.

4. Dini FL, Buralli S, Bajraktari G, Elezi S, Duranti E, Metelli MR, Carpi A, Taddei S. Plasma matrix metalloproteinase-9 better predicts outcome than N-terminal protype-B natriuretic peptide in patients with systolic heart failure and a high prevalence of coronary artery disease. Biomed Pharmacother. 2010; 64(5):339-342.

5. Clements IP, Wiseman GA, Hodge DO, Jacobson AF. Outcome prediction in heart failure with atrial fibrillation: relative role of left ventricular ejection fraction and neurohormonal measures. J Nucl Cardiol. 2013; 20(5):821829.

6. Poulsen SH. Clinical aspects of left ventricular diastolic function assessed by Doppler echocardiography following acute myocardial infarction. Dan Med Bull. 2001; 48(4):199-210.

7. Akincioglu C, Berman DS, Nishina H, Kavanagh PB, Slomka PJ, Abidov A, Hayes S, Friedman JD, Germano G. Assessment of Diastolic Function Using 16-Frame 99mTcSestamibi Gated Myocardial Perfusion SPECT: Normal Values. J Nucl Med. 2005; 46(7):1102-1108.

8. French BA, Kramer DG. Mechanisms of Post-Infarct Left Ventricular Remodeling. Drug Discov Today Dis Mech. 2007; 4(3):185-196.

9. Konstam MA, Kramer DG, Patel AR, Maron MS, Udelson JE. Left Ventricular Remodeling in Heart Failure. J Am Coll Cardiol Img. 2011; 4(1):98-108.

10. Fan D, Takawale A, Lee J, Kassiri Z. Cardiac fibroblasts, fibrosis and extracellular matrix remodeling in heart disease. Fibrogenesis Tissue Repair. 2012; 3; 5(1):15.

11. Hillis GS, Møller JE, Pellikka PA, Gersh BJ, Wright RS, Ommen SR, Reeder GS, Oh JK. Noninvasive estimation of left ventricular filling pressure by e/e' is a powerful predictor of survival after acute myocardial infarction. J Am Coll Cardiol. 2004; 43(3):360-367.

12. Pinamonti B, Zecchin M, Di Lenarda A, Gregori D, Sinagra G, Camerini F. Persistence of Restrictive Left Ventricular Filling Pattern in Dilated Cardiomyopathy: An Ominous Prognostic Sign. J Am Coll Cardiol Img. 1997; 29(3):604612.

13. Graham HK, Horn M, Trafford AW. Extracellular matrix profiles in the progression to heart failure. European Young Physiologists Symposium Keynote Lecture-Bratislava 2007. Acta Physiol (Oxf). 2008; 194(1):3-21.

14. Yamazaki T, Lee JD, Shimizu H, Uzui H, Ueda T. Circulating matrix metalloproteinase-2 is elevated in patients with congestive heart failure. Eur J Heart Fail. 2004; 6(1):41-45.

15. George J, Patal S, Wexler D, Roth A, Sheps D, Keren G. 
Circulating matrix metalloproteinase-2 but not matrix metalloproteinase-3, matrix metalloproteinase-9, or tissue inhibitor of metalloproteinase-1 predicts outcome in patients with congestive heart failure. Am Heart J. 2005; 150(3):484-487.

16. Rossi A, Cicoira M, Golia G, Zanolla L, Franceschini L, Marino P, Graziani M, Zardini P. Amino-terminal propeptide of type III procollagen is associated with restrictive mitral filling pattern in patients with dilated cardiomyopathy: a possible link between diastolic dysfunction and prognosis. Heart. 2004; 90(6):650-654.

17. Cicoira M, Rossi A, Bonapace S, Zanolla L, Golia G, Franceschini L,Caruso B, Marino PN, Zardini P. Independent and additional prognostic value of aminoterminal propeptide of type III procollagen circulating levels in patients with chronic heart failure. J Card Fail. 2004; 10(5):403-411.

18. Schiller NB, Shah PM, Crawford M, DeMaria A, Devereux R, Feigenbaum H, Gutgesell H, Reichek N, Sahn D, Schnittger I. Recommendations for quantitation of the left ventricle by two-dimensional echocardiography. American Society of Echocardiography Committee on Standards, Subcommittee on Quantitation of Two-Dimensional Echocardiograms. J Am Soc Echocardiogr. 1989; 2(5):358367.

19. Muntinga HJ, van den Berg F, Knol HR, Niemeyer MG, Blanksma PK, Louwes H, van der Wall EE. Normal values and reproducibility of left ventricular filling parameters by radionuclide angiography. Int J Card Imaging. 1997; 13(2):165-171; discussion 173.

20. Pencina MJ, D’Agostino RB Sr, D'Agostino RB Jr, Vasan RS. Evaluating the added predictive ability of a new marker: from area under the ROC curve to reclassification and beyond. Stat Med. 2008; 27(2):157-172; discussion 207-112.

21. Steyerberg EW, Vickers AJ, Cook NR, Gerds T, Gonen M, Obuchowski N, Pencina MJ, Kattan MW. Assessing the performance of prediction models: a framework for traditional and novel measures. Epidemiology. 2010; 21(1):128-138.

22. Pencina MJ, D'Agostino RB. Overall $\mathrm{C}$ as a measure of discrimination in survival analysis: model specific population value and confidence interval estimation. Stat Med. 2004; 23(13):2109-2123.

23. Kim HE, Dalal SS, Young E, Legato MJ, Weisfeldt ML, D'Armiento J. Disruption of the myocardial extracellular matrix leads to cardiac dysfunction. J Clin Invest. 2000; 106(7):857-866.

24. Alla F, Kearney-Schwartze A, Radauceanu A, Das Dores S, Dousset B, Zannad F. Early changes in serum markers of cardiac extra-cellular matrix turnover in patients with uncomplicated hypertension and type II diabetes. Eur J Heart Fail. 2006; 8 (2):147-153.

25. Lin $\mathrm{YH}$, Lin C, Lo MT, Lin HJ, Wu YW, Hsu RB, Chao CL, Hsu HC, Wang PC, Wu VC, Wang SS, Lee CM,
Chien KL,et al. The relationship between aminoterminal propeptide of type III procollagen and heart rate variability parameters in heart failure patients: a potential serum marker to evaluate cardiac autonomic control and sudden cardiac death. Clin Chem Lab Med. 2010; 48(12):18211827.

26. Ban CR, Twigg SM. Fibrosis in diabetes complications: Pathogenic mechanisms and circulating and urinary markers. Vasc Health Risk Manag. 2008; 4(3):575-596.

27. Cavallari LH, Groo VL, Momary KM, Stamos TD, Vaitkus PT. Markers of cardiac collagen turnover are similar in patients with mild and more severe symptoms of heart failure. Congest Heart Fail. 2007; 13(5):275-279.

28. Hung CS, Chou $\mathrm{CH}, \mathrm{Wu} \mathrm{XM}$, Chang YY, Wu VC, Chen YH, Chang YS, Tsai YC, Su MJ, Ho YL, Chen MF, $\mathrm{Wu} \mathrm{KD}$, Lin YH. Circulating tissue inhibitor of matrix metalloproteinase-1 is associated with aldosterone-induced diastolic dysfunction. J Hypertens. 2015; 33(9):1922-1930; discussion 1930.

29. Hung CS, Chou CH, Liao CW, Lin YT, Wu XM, Chang YY, Chen YH, Wu VC, Su MJ, Ho YL, Chen MF, Wu $\mathrm{KD}$, Lin YH. Aldosterone Induces Tissue Inhibitor of Metalloproteinases-1 Expression and Further Contributes to Collagen Accumulation: From Clinical to Bench Studies. Hypertension. 2016; 67(6):1309-1320.

30. Frantz S, Stork S, Michels K, Eigenthaler M, Ertl G, Bauersachs J, Angermann CE. Tissue inhibitor of metalloproteinases levels in patients with chronic heart failure: an independent predictor of mortality. Eur J Heart Fail. 2008; 10(4):388-395.

31. Chang YY, Chen A, Wu XM, Hsu TP, Liu LY, Chen YH, Wu YW, Hsu RB, Lee CM, Wang SS, Lo MT, Chen MF, Lin YH. Comparison the Prognostic Value of Galectin-3 and Serum Markers of Cardiac Extracellular Matrix Turnover in Patients with Chronic Systolic Heart Failure. Int J Med Sci. 2014; 11(11): 1098-1106.

32. Wong M, Staszewsky L, Latini R, Barlera S, Glazer R, Aknay N, Hester A, Anand I, Cohn JN. Severity of left ventricular remodeling defines outcomes and response to therapy in heart failure: Valsartan heart failure trial (ValHeFT) echocardiographic data. J Am Coll Cardiol. 2004; 43(11):2022-2027.

33. Solomon SD, Anavekar N, Skali H, McMurray JJ, Swedberg K, Yusuf S, Granger CB, Michelson EL, Wang D, Pocock S, Pfeffer MA. Influence of ejection fraction on cardiovascular outcomes in a broad spectrum of heart failure patients. Circulation. 2005; 112(24):3738-3744.

34. Iwahashi N, Kimura K, Kosuge M, Tsukahara K, Hibi K, Ebina T, Saito M, Umemura S. E/e' two weeks after onset is a powerful predictor of cardiac death and heart failure in patients with a first-time ST elevation acute myocardial infarction. J Am Soc Echocardiogr. 2012; 25(12):12901298.

35. Akkan D, Kjaergaard J, Møller JE, Hassager C, TorpPedersen C, Køber L. Prognostic importance of a short 
deceleration time in symptomatic congestive heart failure. Eur J Heart Fail. 2008; 10(7):689-695.

36. Temporelli PL, Corrà U, Imparato A, Bosimini E, Scapellato F, Giannuzzi P.. Reversible restrictive left ventricular diastolic filling with optimized oral therapy predicts a more favorable prognosis in patients with chronic heart failure. J Am Coll Cardiol. 1998; 31(7):1591-1597.

37. Duncan AM, Lim E, Gibson DG, Henein MY. Effect of dobutamine stress on left ventricular filling in ischemic dilated cardiomyopathy: pathophysiology and prognostic implications. J Am Coll Cardiol. 2005; 46(3):488-496.
38. Westenberg JJ. CMR for Assessment of Diastolic Function. Curr Cardiovasc Imaging Rep. 2011; 4(2):149-158.

39. Boxt LM. Evaluation of left ventricular diastolic function with cardiac MR imaging. Invited commentary. Radiographics. 2011; 31(1):259-261. 